\title{
Research on Prevention of Tennis Injury in Various Ages in Universities
}

\author{
Xin Wei \\ Jilin Agricultural University, Changchun 130118, Jilin, China \\ 1005488505@qq.com
}

Keywords: Tennis; Different groups; Sports injuries; Preventive measures

\begin{abstract}
With the continuous improvement of people's living standards, tennis is becoming more and more popular. Tennis is a sport that can make people healthy and passionate. So many people began to know tennis, and had a strong interest in it. And also more and more people began to contact with tennis. However, due to the lack of understanding of the tennis, non-standardized actions and other factors, some people in practice not only did not play the benefits of tennis on people, but also caused their own sports injuries. In this paper, through the research of literature, questionnaire, mathematical statistics and other scientific research methods, the characteristics and laws of sports injury in different tennis groups in colleges and universities are investigated and analyzed to find out the various influencing factors of sports injury, and then understand the basic situation of the causes of sports injuries, so as to prevent and reduce the occurrence of sports injuries in tennis, and put forward effective preventive measures. The purpose of this study is to allow the majority of tennis enthusiasts and their participants to better understand and master the knowledge of tennis injury prevention, to deepen the understanding of tennis, improve self-protection awareness and try to avoid the process of tennis damage, so that people can better enjoy the fun of tennis. The significance of this study is to allow tennis enthusiasts to improve self-protection awareness and have corresponding preventive measures to those body parts which easily cause damage.
\end{abstract}

\section{Introduction}

Selection Basis. With the continuous improvement of people's living standards, tennis is becoming more and more popular [1]. Tennis is a sport that can make people healthy and passionate. So many people began to know tennis, and had a strong interest in it [2]. And more and more people began to contact with tennis. However, due to the lack of understanding of the tennis, non-standardized actions and other factors, some people in practice not only did not play the benefits of tennis on people, but also caused their own sports injuries [3-5]. Therefore, the purpose of this study is to allow tennis enthusiasts to better understand and master the knowledge of tennis injury prevention, to deepen the understanding of tennis, to improve self-protection awareness, and to try to avoid injury, so that people can better enjoy the fun of tennis and experience the joy of sports.

Research Status. Sports injury refers to the various injuries that occur in the process of sport [6]. Its occurrence is related to sports training arrangements, sports and technical action, sports training level, sports environment and conditions and other factors. Sports injury, as one of the main research areas of clinical practice of sports medicine, has laid a reliable theoretical foundation for the development of sports medicine, which has become a characteristic of sports science research and shows a wide range of research prospects. Here, this paper summarizes the current situation and development of tennis injury research in terms of the causes of sports injuries and the preventive measures. At present, China's research in this field is mostly for the prevention of occupational injury of professional athletes, tennis elbow, shoulder joint and other occupational injuries. It elaborated the principle of damage and put forward the corresponding preventive measures.

\section{Research Objects and Methods}

Research Object. The people of different ages in ordinary college who participate in tennis [7]. That is, 
five age groups, each group has fifteen men and fifteen women. The details are shown in Tab .1. Table 1. Statistics of tennis crowd in different age in ordinary colleges

\begin{tabular}{llll}
\hline Under 18 years old & 30 & 15 & 15 \\
$19-25$ years old & 30 & 15 & 15 \\
$26-35$ years old & 30 & 15 & 15 \\
$36-50$ years old & 30 & 15 & 15 \\
Over 50 years old & 30 & 15 & 15 \\
\hline
\end{tabular}

Research Methods. The first one is the documentation method. According to the needs of this paper, I have checked the relevant materials, and added my own several years of experience to analyze and study. The second one is the questionnaire survey method. According to the needs of this subject, a questionnaire on the sports injury of tennis people in different ages was designed. Questionnaire issued 150 copies, withdrew 150 , the utilization rate is $100 \%$. The third one is the mathematical statistics method. We summarize and sort out some materials and questionnaires that have been collected and count and analyze the data.

\section{Results and Discussion}

Results.

Table 2. Probability Statistics of Age and Injured parts

\begin{tabular}{cccccc}
\hline $\begin{array}{c}\text { AgelProbability } \\
\text { of injured parts }\end{array}$ & $\begin{array}{c}\text { Shoulder } \\
\text { joint }\end{array}$ & Elbow joint & Wrist joint & Ankle joint & Other joints \\
\hline $\begin{array}{c}\text { Under 18 years } \\
\text { old }\end{array}$ & 26.5 & 24.6 & 16.7 & 7.8 & 24.4 \\
$19-25$ years old & $27.4 \%$ & $30.3 \%$ & $14.7 \%$ & $8.8 \%$ & $18.8 \%$ \\
26 -35 years old & $23.6 \%$ & $29.5 \%$ & $18.9 \%$ & $8.6 \%$ & $19.4 \%$ \\
36 -50 years old & $24.7 \%$ & $28.9 \%$ & $17.3 \%$ & $7.7 \%$ & $21.4 \%$ \\
Over 50 years old & $30.5 \%$ & $27.4 \%$ & $13.6 \%$ & $6.8 \%$ & $21.7 \%$ \\
\hline
\end{tabular}

From Tab .2, we can see that different groups of people in the process of playing tennis have different body injury risks, but in addition to the impact of age factors, there are also many other factors. Therefore, I count the results of my own questionnaires and other questions, which are as follows:

$62.4 \%$ people injure because of the insufficient preparation and informal technical action; Shoulder and elbow joint injury accounted for $54.5 \%$, which are higher than the risk of other joint injuries; $53.4 \%$ of the older people injure in the process of playing tennis, which are more than those of the younger age. The people of different ages have different injuries in the process of playing tennis. For the people who participated in tennis for a long time, their chance of injury is $38.6 \%$, which is less than the people who participated in tennis for a short time. For the people who not often play tennis, their chance of injury is $62.6 \%$, which is greater than the people who often participate in tennis. The people who do not often participate in other sports, their chance of injury accounted for $59.2 \%$, which is greater than those who often participate in other projects; The probability of exercise injury in winter accounted for $68.6 \%$, which is greater than the other seasons; For male, risk of injury accounted for $66.4 \%$, which is greater than the chance of injury of women.

Discussion. According to the questionnaire results: in the process of tennis, before the activities, athletes don't have a sufficient preparation and their technical action is not standardized so it is very easy to cause injury. Most people's shoulder and elbow joints are more easily injured. The older people's injury probability is much higher than the younger people. As the people who participate in tennis are at different ages, there are different parts of the movement injury during the course of tennis. Those who do not often participate in sports are more likely to suffer from sports injuries than those who regularly participate in sports. As the season changes, in winter, bodies heat slowly, and the joints of the bodies are in a hibernation state, so the probability of winter sports injury is much larger than the other season. So we can see the importance of sports on the human body. More exercise and more health. 
Sports injury is an important part of sports medicine. It studies the prevention, treatment and rehabilitation of sports injuries. By summarizing the causes, treatment effect and rehabilitation time of sports injuries, it provides the scientific basis and guidance for the improvement of the exercise conditions, teaching and training methods and sports results.

The first one of the discussion is that during the course of tennis, several injuries and causes are likely to occur:

From the results of the questionnaire, we can be see that in the process of tennis, the age of the most prone to movement damage is the shoulder and elbow joint. The two parts of the probability of injury accounted for about half of the overall, for which I consult some literature materials and summarize the causes of the damage:

Tennis elbow reasons: People suddenly stretch the tendon near the elbow, so that it is embedded in the bone on the ankle. Mostly the action that using the backhand to hit the ball is not correct or the racket is too heavy so that it cause forearm muscle tension, or elbow inflammation. In addition, the recent foreign scholars also suggested: If the forearm muscle strength, toughness and endurance are in a bad situation, coupled with technical action errors, especially anti-shot technology action error, it will be also prone to tennis elbow. Symptoms: elbow pain, and then become the whole arm pain.

The reasons of shoulder joint pain: shoulder joint muscles, ligaments and joint capsule have heavy load, coupled with excessive force caused by the service. Symptoms: Shoulder joint hurts when people serve, hit the ball and do the smash, and the arm cramps. The relevant materials show that the shoulder pain of tennis is often the disease of rotator cuff caused by repeated internal load. The coracoacromial arch stenosis of young athletes is the reactivity change caused by the upwards shift impact of caput humeri. The primary factor is: The first one is the centrifugal load of the supraspinatus tendon in acceleration period, throwing moment and deceleration period. The second one is the physiological weakness and chemical inflammation caused by fatigue. The third one is that the vascular damage, permanent tendon changes, frequent tear process and pathological changes lead to physiological and structural weakness. The fourth one is that the humeral head is out of control and moved upward. Rotator cuff is a key factor in the control of the humeral head. The fifth one is the labrum injury. The sixth one is the secondary coracoacromial arch impact. The seventh one is that acromioclavicular arthritis often associated with rotator cuff tendonitis.

For the young athletes of tennis, fibrosis and osteophyte are possible. For the 50 and 60 years old recreational athletes, there may be the subacromial change that are related to the above, but it is secondary. This conclusion was confirmed by NEER in 1972 in the classic report. In this report, only $25 \%$ patients whose rotator cuffs tear fully have the subacromial change. Older athletic athletes are more likely to form the full rotator cuffs tear. And the upwards shift of most brachial bone is caused by this factor [8].

The second one of the discussion is the classification of sports injuries. According to the damage situation of people in different ages during the above-mentioned tennis movement, the sports damage of tennis sports is divided into the following categories:

According to the injured organizational structure: such as skin damage, muscle and tendon injury, joint injury, bursa injury, bone injury, epiphyseal injury, nerve injury and internal organs injury. According to the post-injury skin or mucosal integrity, it is divided into open injury and closed injury. For open injury, the integrity of injury skin or mucosa is destroyed and there is a wound that connected with the outside world. Such as abrasions, stab wounds, laceration and open fractures. For closed injury, injury skin and mucosa remains intact and there is no wound that connected with the outside world. Such as contusion, muscle strain, joint injury, tenosynovitis and closed fractures [9]. (It is the most vulnerable sport injury in tennis)

According to the severity of injury, it is divided into slight wound, medium injury and serious injury. After the slight injury, athletes can be trained as the original plan. After the medium injury, athletes can't be trained as the original plan. They should obey the injury training rules and reduce the injury part movement. For the serious injury, they can't participate in the training.

According to the degree of injury, it is divided into acute injury and chronic injury. Acute injury refers to an injury that is caused by direct and indirect violence. Chronic injury refers to the local 
overload, strain caused by repeated damage or the old injury caused by the improper handling of the acute injury.

The third one of the discussion is the cause of injury. According to the results of the questionnaire, the vulnerable parts of tennis sports, different injury parts and characteristics of different crowd, I will summarize the causes of sports injuries in tennis which are as follows: The first one is that athletes didn't follow the principles of gradual progress in training. When athletes forced themselves to complete some of the exercises that can't be completed or in the process of completing complex technology, they can't control themselves, then they will sprain or fall down. The second one is that in the sports training or competition, the inaccurate technical action will increase the burden on the local body, which is very likely to cause injury. The third one is that in the completion of a technical action, because of the lack of local muscle strength, weak muscle fibers, especially the small muscle groups which are lack of training, people will be hurt easily. The fourth one is the poor flexibility. In sports training and competition, due to the large intensity training and the increase of action range, some muscles and ligaments with poor extensibility may be hurt easily. The fifth one is that the inattention in training and competition is also likely to cause injury. The sixth one is that when the body is in a state of fatigue, continuing to engage in difficult practice is also likely to cause injury. The seventh one is that after a heavy load practice, muscles are in a relatively stiff state. If people are still engaged in large intensity, fast and violent activities, it is easy to strain the muscles. The eighth one is that if training content and exercise arrangements are not good and people engaged in a single training content for a long time, it will also cause local over-limit load and injury. The ninth one is that uneven sites. The tenth is the violation of human physiological anatomy characteristics in practice. For example, around the knee joint, there is little muscle protection. When we squat, the joint ligament will be relaxing, and this will weaken the ability to protect the joints. Once the action isn't correct, people will easily get injured. For another example, the range of motion of cervical vertebra joint is large. In the landing of Fosbury Flop, we should pay attention to prevent sprains and dislocation. The scapular glenoid of shoulder joint is small, but the humeral head is large, and the range of activities is very big. Joint stability depends on the maintain of muscles around the shoulder. When we do the throw practice, the shoulder burden is heavy, we should strengthen the strength practice, flexibility exercises and pay attention to reasonable technology, especially javelin athletes should more pay attention to this [10]. The eleventh one is that the inadequate preparation activities and improper content are the main causes of some sports injuries. The twelfth one is the poor organizational discipline and training order.

The fourth one of the discussion is the principles of exercise injury prevention. Tennis can make people fit and cultivate sentiment, but sports injuries will bring people unnecessary trouble and pain. Therefore, in the process of tennis, for all ages, prevention is more important than treatment, so we must pay attention to the prevention of sports injuries. According to consultation of literature materials and my own years of playing experience, I will put forward some principles on the prevention of tennis sports injury: the first one is to actively carry out the publicity and education work on how to prevent sports injury. The second one is to strengthen the overall physical training, improve the ability to adapt to the movement. It is a positive way to prevent sports injuries by conducting targeted training and improving the function of the vulnerable parts of tennis. The third one is to do reasonable arrangements for the amount of exercise and play time, and do seriously preparation activities. The appropriate internal between the preparation activities and the formal play is 1 to 4 minutes. Generally it is enough to make the body heat and slightly sweat. In winter, we should pay attention to the local burden of sports organs and the training arrangements after injury to prevent local heavy burden. Obey the rules of the competition. The fourth one is to strengthen the protection of movement. Appropriate protection and help in the movement can enhance the confidence of athletes and avoid the occurrence of some accidents. Participants in tennis should learn how to protect themselves. The fifth one is to strengthen the supervision of obligations, establish and improve the self-supervision system to strictly enforce the inspection of the site. Do not wear unsuitable clothes to play tennis.

In short, before exercising, warming-up should be done fully. In actual work, we find that a lot of exercise injuries are caused by the inadequate preparation. Therefore, it is necessary to do a warming-up before exercising. Warming-up can improve the excitability of the central nervous system, overcome the 
physiological inertia of the functional activities of the body, and prepare for formal exercises. Warming-up can increase the number of open capillaries in muscle, improve muscle strength, elasticity and flexibility, and also improve the function of joint ligament and enhance the ligament flexibility, so as to increase the intra-articular synovial fluid and prevent muscle and ligament injury. During the warming-up, both trunk and limbs' large muscle groups and joints should be moved fully, and also all the small joints should be moved.

The interval relaxation also should be paid attention. In exercise, in order to eliminate the muscle fatigue quickly and prevent movement injuries due to partial overload, it is important to relax between exercises. In the interval, some athletes don't pay enough attention to this problem. After each exercise, they often stand aside or monotonously do some relaxation exercises. In that way, they can't speed up the elimination of fatigue, but hurt themselves. As the content of each program is different, the form of internal relaxation should also be different. For example, for the program which focuses on the upper limb exercises, we can do some relaxing jogging in the interval. For the program which focuses on the lower limbs, after the exercise, we can lie on our back on the mat or grass and then lift the legs to shake or stand upside down [5]. This can promote blood return, improve the blood supply, and also enhance the suppression of the nerve cells which have fatigued and let them get a rest. It has positive significance for eliminating fatigue and preventing sports injury.

Local overload should be avoided. Excessive concentration of exercise during exercise can cause local overload and sports injuries. Therefore, in exercise, we should avoid monotonous and one-sided training methods to prevent partial overload. In addition to the above several, we also should pay attention to sports protection and the selection of exercise site. It is also an important part of preventing sports injury.

\section{Conclusion}

The impact of sports injuries is serious. It makes the athletes can not participate in the normal training and competition, so as to hinder the improvement of athletic performance. It also shortens the athletes' sports life. Severe cases can also cause disability, or even death. For the general tennis enthusiasts, sports injuries will affect their health, learning and work, cause adverse psychological impact, and hinder the normal movement of sports.

Prevention of tennis injuries is more important than treatment. As long as we have a good understanding of the significance of prevention of sports injuries, carefully investigate and study, timely sum up lessons, master the law of the occurrence of sports injuries and do preventive work, we can minimize or avoid sports injuries, so as to better participate in tennis, and make the movement play its true nature of physical fitness and influencing sentiment.

\section{References}

[1] Z.D.Guo and H.Zhang: Modern Preventive Medicine, Vol.39(2012), No.1, p.93. (In Chinese)

[2] J.C.Wang: Journal of Luohe Technology College, Vol.11(2012), No.2, p.181. (In Chinese)

[3] Y.Zhu, J.K.Yang and H.W.Zhu: Guizhou Sports Science and Technology, 2013, No.4, p.56. (In Chinese)

[4] K.K.Wang: Guizhou Sports Science and Technology, 2013, No.4, p.62. (In Chinese)

[5] H.J.Li and H.X.Yao: Journal of Inner Mongolia Medical University, Vol.35 (2013), No.6, p.497. (In Chinese)

[6] F.Song: Gansu Science and Technology, Vol.29 (2013), No.18, p.73. (In Chinese)

[7] L.Wang: Contemporary Sports Technology, Vol.4 (2014), No.31, p.119. (In Chinese)

[8] F.F.Tan and D.F.Zhang: Sports World, 2015, No.9, p.130. (In Chinese)

[9] G.S.Wang: Journal of Langfang Teachers University, Vol.16 (2016), No.1, p.110. (In Chinese)

[10] J.Zhao: Contemporary Sports Technology, Vol.7 (2017), No.6, p.19. (In Chinese) 